\section{Colour Chemigrams}

Siegfried Manietta, Photography, Queensland College of Art, Griffith University, PO Box 3370 South Brisbane QLD 4101 Australia. E-mail:

$<$ s.manietta@griffith.edu.au>.

See < www.mitpressjournals.org/toc/leon/45/3> for supplemental files associated with this issue.

\section{Submitted: 12 July 2010}

\section{Abstract}

This paper describes an original colour chemigram technique utilizing couplers incorporated in fully processed traditional colour photographic materials through reaction with selectively oxidized colour developing agents. Because this process bypasses light-sensitive silver halides, image structure is essentially molecular in scale and the procedure is performed under normal room illumination. Images of remarkable beauty have been produced from discarded colour films and papers. Notionally the term "chromagram" is proposed for this procedure.

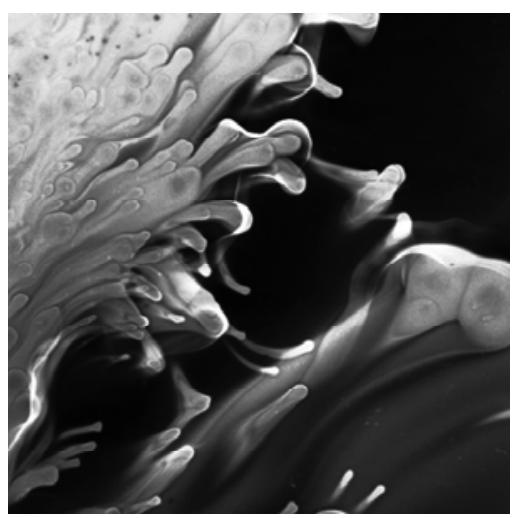

Fig.1. Untitled Chromagram (detail), $5 \times 4$ in, Kodak Vericolor Internegative Film. ( S. Manietta)

The expression "chemigram" is attributed to Pierre Cordier (1958) [1]. The term generally refers to the use of blackand-white photochemistry to create abstract images on black-and-white photographic papers in subdued room light. Image colour is usually, though not exclusively, generated due to complex oxidation-reduction byproducts and local physical development effects.

Working in an educational environment I am frequently confronted by extraordinary waste in the form of discarded colour materials: out-of-date films, film unprocessed and exposed before loading, clear film processed without an image, totally clear colour transparencies, unexposed white cleaning paper issuing from a roller-transport processor. Converting these materials into chemigrams not only recycles waste but also opens creative windows. With a minimum of technical skill, a little care and patience, photographic artists can create exquisite colour images using proprietary materials and commonly available photochemistry.

\section{Background}

Contemporary chromagenic (colour forming) photographic materials function on chemical principles pioneered in 1912 by Rudolph Fischer [2], who discovered that the oxidized form of the developing agent paraphenylenediamine (PPD) reacted with complex chemicals termed colour couplers or colour formers.

Fischer's experiments can be replicated by preparing a colour developer solution (any colour developer) containing the coupler $\alpha$-napthol at approximately 1 gram per litre: if the mixture is allowed to stand for a few minutes, a thin surface layer of blue dye forms in the wake of aerial oxidation. Chemically oxidizing the developer by developing some B\&W paper creates a blue dye within the emulsion. Adding a small quantity of concentrated potassium ferricyanide solution, for example, instantly turns the entire mixture dark blue. In modern colour processes, this reaction and attendant colour formation are tightly controlled. The chromagenic development reaction is generally written in a simplified twostep form:

Step 1. Exposed Silver Halide $+\mathrm{Col} \mathrm{Dev} \rightarrow$ Silver (metal) + Halogen + Oxidized Col Dev Step 2. Oxidized Col Dev + Colour Coupler $\rightarrow$ Dye

In commercial chromagenic processes the metallic silver remaining after the developing agent has oxidized and coupled to form dye is removed from the emulsion, as this would degrade image colour [3]

Fully processed colour emulsions therefore contain dye - formed by coupling in the exposed and developed regions - and unused colour couplers that surprisingly remain reactive within the emulsion for the life of the image.

\section{Photographic Materials}

Contemporary colour materials must be capable of analyzing a projected image in three components: red, green and blue - this is accomplished by layering a minimum of 3 emulsions, each responsive to one of the three primary spectral bands hence their historical name "tripack" films.

Film designed for camera exposure (including "laboratory" films such as internegative films, print films and duplicating films) employs a layer structure with yellow-forming couplers on top of magenta- and cyan-forming couplers. Photographic paper and some printing films have the cyan layer above to improve resolution and fade resistance. Layer structure essentially determines the range and location of colour formed. Reversal films, print films and internegative films generally produce more saturated colour images due to increased layer thickness and incorporated coupler density.

Because silver halides are bypassed in this procedure, out-of-date film, processed or not, generally works well. Pre-treatment with farmer's reducer, bleach-fix or separate bleach and fix is required for unprocessed film to clear silver compounds and sensitizing dyes. Pre-treatment processing conditions are essentially inconsequential except that silver and silver compounds must be removed. The richest source of material is either unexposed processed colour negative film from the local lab or totally fogged and processed (clear) transparency film. Essentially film that customers didn't bother taking home!

\section{Developers}

Any working strength proprietary colour developing solution may be used as all are derived from paraphenylenediamine (PPD). Dye yield and colour shift are possible with some coupler/developing agent combinations; however, this is inconsequential, as the images formed are not pictorial.

\section{Oxidizing Agents}

The developer oxidizing agents tested for this work were selected from traditional photochemistry stock and included potassium permanganate, potassium ferricyanide, hydrogen peroxide and ammonium dichromate. Some strong oxidizing agents, such as potassium permanganate, applied in crystalline form directly to moist emulsions, were found to damage the gelatin structure (probably due to surface tanning effects), creating fine emulsion pinholes. 


\section{Technique}

Simply adding any pre-oxidized colour developer to an emulsion containing dispersed colour couplers will generate dye in each layer - sequentially - as solution is absorbed by the gelatin. Preoxidizing commercially formulated colour developers is not as straightforward as one might imagine, as these preparations contain substantial quantities of anti-oxidants.

I found that pre-soaking an emulsion in colour developer (collected from the overflow of a colour paper processor), draining excess surface solution and adding concentrated oxidizing agent locally induced virtually instantaneous coupling at the point of contact, before antioxidants could protect the developing agent.

Syringes were found useful for applying the small quantities of chemicals required. Practical variations included pre-soaking an emulsion in either an oxidizing solution or the developer and selectively applying either the developer or the oxidizing agent. Each method of application has potentially different image outcomes depending upon whether developer or oxidizing agent was available in excess and local solution flow dynamics.

One particularly interesting method involves placing developer-soaked fibrous tissue in contact with an emulsion and sprinkling potassium ferricyanide crystals over the tissue. The ferricyanide oxidizes the developer while rapidly absorbing water from the developer solution, causing the paper to shrink, forming capillary channels that create flow patterns of remarkable beauty and intricacy.

Flowing a small quantity of developer around crystallized oxidizing agent on the surface of a film or paper "autographically" records eddies and flow patterns. Placing an object prepared with oxidizing agent on an emulsion infused with developer creates an autographic imprint.

Once the desired effect has been achieved, a 5-10 minute wash in clean running water at ambient temperatures completes the process, as there is nothing to "fix". The procedure may be repeated until all available couplers are converted to dye and the emulsion reaches maxi-

mum black. Post-treatment with proprietary C-41 or E-6 stabilizing solution may be considered where images are deemed worth conserving.

\section{Influencing Image Colour}

Dye formation by oxidized developer in camera films follows the layer structure described earlier and occurs first in the top yellow forming layer, then proceeds to the magenta and cyan layers. Original appear dominantly yellow, green, black and clear where coupling did not occur. When such an image is printed using standard neg-pos enlarging or contact printing methods, colours graduate from blue to green to white. Where a paper emulsion is employed the gradation is from cyan (top layer) to magenta to yellow. Originals appear dominantly cyan, blue, black and clear where coupling did not occur. Colours formed in neg-pos printing on paper graduate from red to yellow to white. Scanned images may of course be printed in any colour. With a little understanding of the process fundamentals and a material's layer structure, the outcomes become reasonably controllable and predictable.

\section{Discussion}

I have exhibited chromagram prints on 3 occasions. All were made using traditional (optical) colour photographic printing from negative images created using this technique. While the process at first sight appears entirely random, sufficient control is possible to generate outcomes with pre-determined aesthetic qualities or "feel". The exhibition $\mathrm{Nou}$ menal Landscapes (2009) used patterns reminiscent of satellite images to infer fantasy places, while Requiem (2008) addressed the emotional highs and lows associated with the experience of death and grief. Ultimately the visual "reading" of these images is entirely dependent upon process dynamics, the artist's intent and the viewer's perceptions.

I have recently conducted further experiments to adjust the colour gamut of the chromagram process. These involve the inclusion of Kodachrome couplers in solution. This has proven useful for counteracting the tendency for the upper layer to generate overall blue in negative to positive prints. Cyan coupler in the developer solution "drives" the primary colour to red/magenta.

Important Health \& Safety Note All colour-developing agents are either known allergens, carcinogens or derived from known allergens and carcinogens. As with any photographic process, adequate ventilation, good housekeeping and careful handling of both developers and oxidizing agents are vital for a safe workplace. Chemicals must be stored in properly labeled bottles, and gloves must be worn while handling these. Material Safety Data Sheets (MSDS) are available from manufacturers' web sites and need to be checked before proceeding.

\section{References and Notes}

1. $<$ www.pierrecordier.com/en/lexique.html $>$. 2. R. Fischer, Ger. 253,335 (1912).

3. Note: An excellent description of photographic colour formation by coupling may be found in: L.K.J. Tong, "Mechanism of Dye Formation and related reactions", in T. H. James, ed., The Theory of the Photographic Process (London: Collier Macmillan Publishers, $4^{\text {th }}$ ed., 1944).
Fig. 2. Chromagram

Requiem - 120 Agfa

Optima 100 colour negative film $6 \times 18$ cm, printed $50 \times 150$ cm. (๑) S. Manietta 2008)

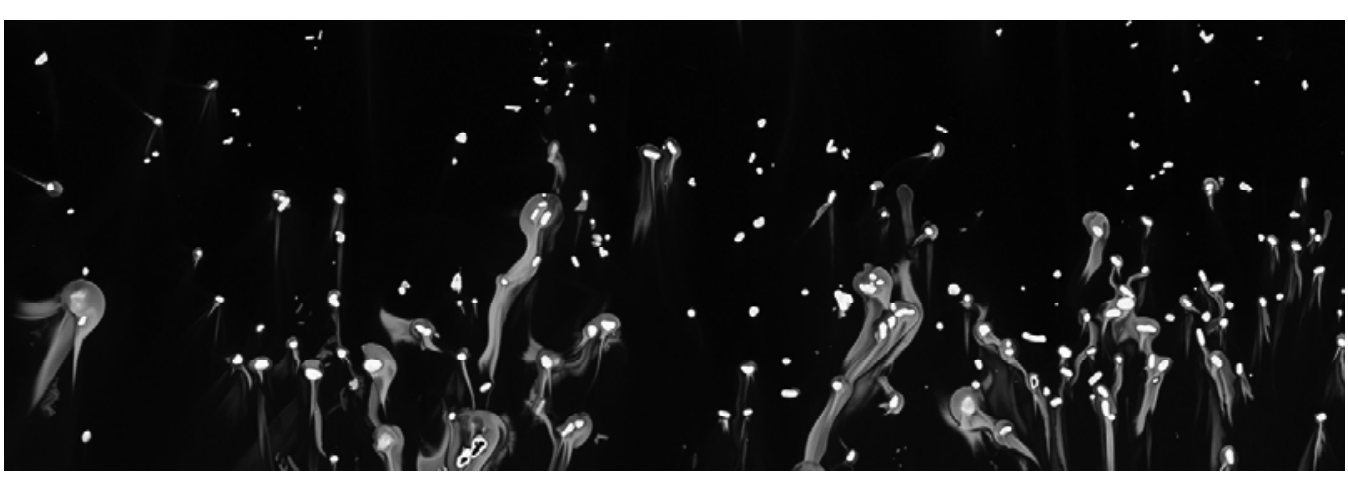

